\title{
The Vascular Basis of the Pronator Quadratus Muscle Flap and Its Use in Clinical Cases
}

\author{
Binu P. Thomas ${ }^{1}$ Sasi P. Kiran ${ }^{1}$ Maolin Tang ${ }^{2}$ Christopher R. Geddes ${ }^{2}$ Steven F. Morris ${ }^{2}$ \\ ${ }^{1}$ Dr Paul Brand Centre for Hand Surgery, Christian Medical College \& \\ Hospital, Vellore, India \\ ${ }^{2}$ Department of Anatomy and Neurobiology and Surgery, Dalhousie \\ University, Halifax, Nova Scotia, Canada

\begin{abstract}
Address for correspondence Dr. Binu P Thomas, D Orth, MS Orth, PDFHS, Prof \& Head, Paul Brand Centre for Hand Surgery, Christian Medical College \& Hospital, Vellore, TN, 632004 India, (e-mail: binu@cmcvellore.ac.in).
\end{abstract}

Indian J Plast Surg:2021;54:63-68.

\begin{abstract}
Background Pronator quadratus (PQ) is a deeply situated muscle in the forearm which may occasionally be utilized for soft-tissue reconstruction. The purpose of this anatomical and clinical study was to confirm vascular supply of PQ muscle (PQM) in order to optimize its transfer and confirm its utility in clinical situations.

Methods In Part A of the anatomical study, fresh human cadavers $(n=7)$ were prepared with an intra-arterial injection of lead oxide and gelatin solution, and PQM and neurovascular pedicle were dissected $(n=14)$. In the anatomical study Part $B$, isolated limbs of embalmed human cadavers $(n=12)$ were injected with India ink-gelatin mixture and PQ were dissected.

Results $P Q$ is a type II muscle flap, with one major pedicle, the anterior interosseous (AI) vessels and two minor pedicles from the radial and ulnar vessels. The mean dimensions of the muscle were $5.5 \times 5.0 \times 1.0 \mathrm{~cm}^{3}$, mean pedicle length was $9.6 \mathrm{~cm}$, and the mean diameter of the artery and the vein was $2.3 \mathrm{~mm}$ and $2.8 \mathrm{~mm}$, respectively. The

\section{Keywords}

- pronator quadrates

- vascular supply of

- free muscle transfer

- composite flap of

- anterior interosseous artery

$-1,2$ ICSRA dorsal cutaneous perforating branch (DPB) of the artery supplied the skin over the dorsal forearm and wrist. This branch also anastomosed with the 1,2 intercompartmental supraretinacular artery (ICSRA).

Conclusion This study confirms the potential utility and vascular basis of the PQM flap and its associated cutaneous paddle.

In the clinical part, two patients with nonhealing wounds exposing the median nerve and flexor tendons in the distal forearm were treated using the PQM flap with good results.
\end{abstract}

\section{Introduction}

Each new surgical procedure or wave of surgical endeavor requires further anatomical information. Understanding the vascular anatomy of individual muscles is critical for muscle transfers, especially with advancements in microsurgical reconstruction. An ideal muscle for transfer is appropriately sized, has constant anatomy, is adequately sized vessels and also expendable. Pronator quadratus muscle (PQM) has many of these characteristics.

The PQ is a small deeply seated expendable muscle of the forearm. The vascular anatomy of $\mathrm{PQ}$ and its use as a

published online September 7, 2020
DOI https://doi.org/

10.1055/s-0040-1716421

ISSN 0970-0358. myo-osseous flap based on the distal blood supply has been reported. ${ }^{1,2}$ It has been used as a pedicled flap to cover the median nerve after neurolysis or excision of neuromas, ${ }^{3}$ as a pedicled myo-osseous flap in the treatment of scaphoid nonunions, ${ }^{4}$ and other conditions, ${ }^{5}$ for coverage of palmar defects, ${ }^{6}$ forearm and wrist defects, ${ }^{7}$ and as a functioning free muscle transfer for thenar muscle loss. ${ }^{8}$ Anatomical studies have also suggested the possibility of using this flap for facial reanimation. ${ }^{9}$

Previous studies have not clarified the relationship between the vascular anatomy of the PQ and surrounding structures. The objectives of this anatomical and clinical

(C) 2020. Association of Plastic Surgeons of India.

This is an open access article published by Thieme under the terms of the Creative Commons Attribution-NonDerivative-NonCommercial-License, permitting copying and reproduction so long as the original work is given appropriate credit. Contents may not be used for commercial purposes, or adapted, remixed, transformed or built upon. (https://creativecommons.org/licenses/by-nc-nd/4.0/)

Thieme Medical and Scientific Publishers Pvt. Ltd. A-12, 2nd Floor,

Sector 2, Noida-201301 UP, India 
study were to (i) define the neurovascular anatomy of the PQ (ii) clarify its intramuscular vascularity, (iii) identify the vascular basis of harvesting pronator quadratus as a pedicled or free flap with skin or bone or both, and (iv) use the PQM flap for pedicled wound coverage of distal forearm defects.

\section{Materials and Methods}

The two-part anatomical study was performed on fresh and preserved human cadavers.

\section{Part A}

Seven fresh human cadavers ( 4 male and 3 female; mean age 72 years) were injected with the modified lead oxide-gelatin injection technique, ${ }^{10}$ originally described by Salmon ${ }^{11,12}$ and Rees and Taylor ${ }^{13}$ and modified by Tang et al. ${ }^{10} \mathrm{~A}$ mixture of $5 \%$ gelatin (300 Bloom) and lead oxide (100\% w/v) solution was warmed to $40^{\circ} \mathrm{C}$ and injected through a catheter placed in the femoral artery. The total injection volume was $26 \mathrm{~mL} / \mathrm{kg}$. The cadavers were then cooled to $4^{\circ} \mathrm{C}$ for 24 hours. The forearm skin and superficial forearm flexor muscles were removed. The PQM with anterior interosseous (AI) artery, vein and nerve, radial and ulnar arteries and their vena comitantes were meticulously dissected and removed $(n=14)$. The perforating branch from the AI artery (AIA) through the interosseous membrane along with the skin on the dorsum of the wrist it supplied was noted. The specimens were then digitally photographed and radiographed. The photographs, dissection notes and radiographs were analyzed and compared.

\section{Part B}

Six embalmed cadavers (2 males and 4 females, mean age 70 years) were also available for the study. The subclavian artery was injected with a mixture of India ink and gelatin. After 24 hours, the specimens were dissected and analyzed in the same manner as in Part A.

\section{Results}

The modified lead oxide-gelatin injected specimens provided detailed information regarding the extramuscular and intramuscular arterial anatomy. The high-resolution angiograms provided detailed information regarding the arterial anatomy.

The pronator quadratus is a quadrilateral-shaped muscle located in the distal third of the forearm with attachments to the radius, ulna and the interosseous membrane. Superficial and deep heads were identified in all specimens. The deep head was thicker than the superficial head. The mean dimension of the composite muscle was $5.5 \times 5.0 \times 1.0 \mathrm{~cm}^{3}$ (-Fig. 1).

In all of the specimens, the anterior interosseous (AI) artery and nerve provided the principal arterial and nerve supply to the PQ. The average length of the pedicle was $9.6 \mathrm{~cm}$ (range $9.0-10.5 \mathrm{~cm}$ ). The mean diameter of the AIA was $2.3 \mathrm{~mm}$ at its entry into PQ (range $2.0-2.8 \mathrm{~mm}$ ). The AI vein accompanied the artery consistently and was usually single (22 of 26 specimens), with a diameter of $2.8 \mathrm{~mm}$. In

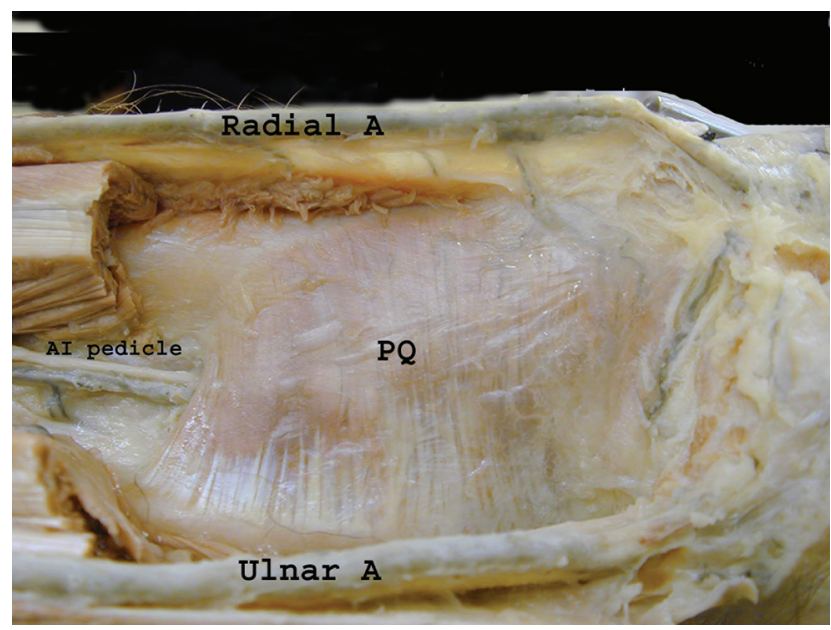

Fig. 1 Photograph of a preserved cadaver dissection specimen with India ink injection showing the pronator quadratus muscle (PQM). The flexor muscle of the forearm has been removed. The radial artery (radial A) and its branches supplying the PQ is clearly seen as is the ulnar artery (ulnar A). The anterior interosseous (AI) pedicle with the artery vein and nerve is seen entering the dorsal aspect of the muscle.

four specimens, there were two venae comitantes ( 4 of 26 specimens). Minor pedicles from radial and ulnar arteries with diameter averaging $0.5 \mathrm{~mm}$ were seen to supply the muscle from radial and ulnar aspects of the muscle, respectively (-Fig. 2). The branches from the radial artery (RA) were identified in all specimens, while those from the ulnar artery (UA) were seen in 20 of the 26 muscles (77\%).

The average length of the AI nerve after the branch to flexor pollicis longus muscle was $4.8 \mathrm{~cm}$ (range $4.0-7.5 \mathrm{~mm}$ ). The AI pedicle entered the dorsal aspect of the muscle in all specimens. At the upper border of the muscle, it gives 1-2 branches to the deep head and then penetrated it to supply the superficial head with 3-4 branches. The radial artery supplied 2-3 branches that entered the radial border of the muscle from the volar aspect ( $\boldsymbol{- F i g . 1}$ ). These branches followed a superficial course for approximately $1 \mathrm{~cm}$ and then penetrated the muscle to anastomose with the branches from the AIA within the superficial head. The branches from the UA when present followed the same pattern. The AI nerve followed the artery and first gave a branch to the deep head and continued distally and volarward to divide into 2-3 terminal branches in order to supply the superficial head.

\section{Dorsal Cutaneous Perforating Branch (DPB) of the Anterior Interosseous Artery (AIA)}

At the proximal border of the muscle, the AIA gave a constant dorsal cutaneous perforating branch (DPB) in all specimens. It pierced the interosseous membrane to supply the skin over the distal third of the forearm and wrist (-Figs. 3, 4). The average diameter of the DPB was $1.2 \mathrm{~mm}$ and the corresponding vein $1.3 \mathrm{~mm}$. It passes between the extensor digitorum communis tendons and the tendons of abductor pollicis longus and extensor pollicis brevis and is located roughly $4 \mathrm{~cm}$ proximal to the Lister's tubercle (LT). The DPB branch divided into two main branches, one of which supplied the skin of the dorsum of the distal forearm and wrist. The 


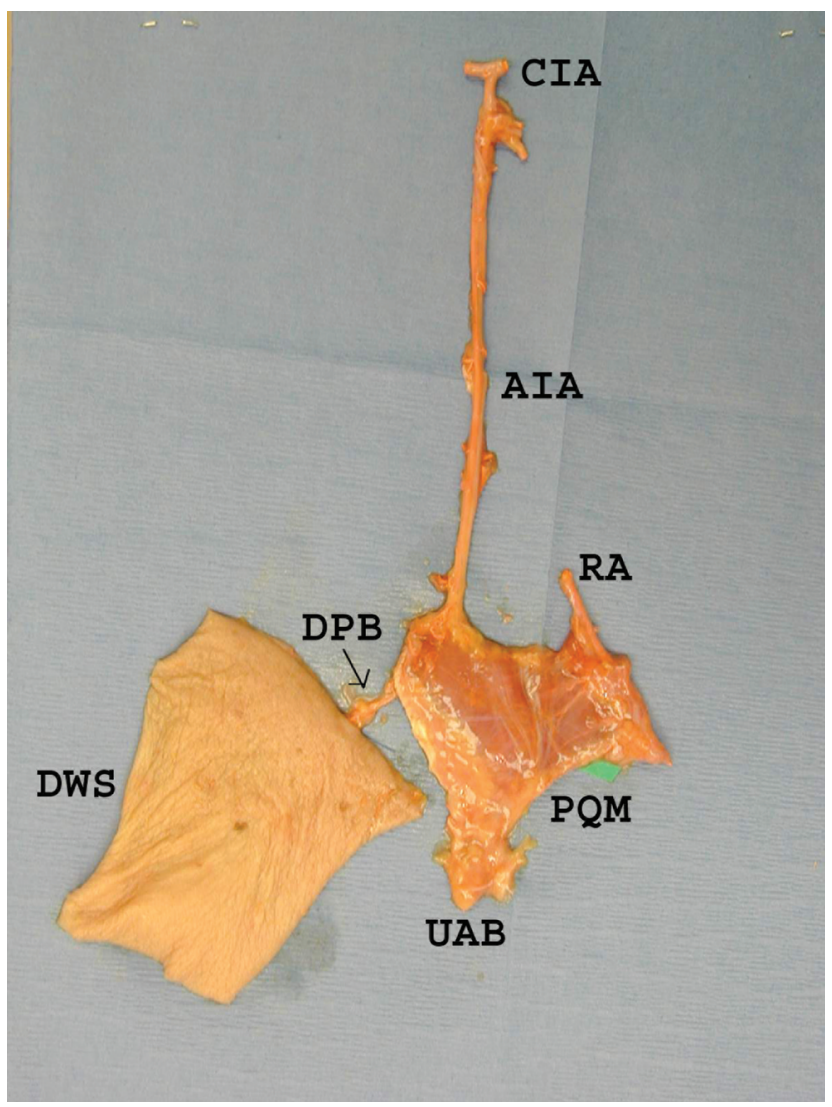

Fig. 2 . Dissected pronator quadratus muscle (PQM) demonstrating the common interosseous artery $(\mathrm{CIA})$ and anterior interosseous artery (AIA) from a lead oxide-gelatin injected fresh cadaver. The radial artery (RA) and ulnar artery (UA) are seen. The dorsal cutaneous perforating branch (DPB) supplying the skin on the dorsum is shown by the arrow.

mean area of skin supplied by this branch was $21 \mathrm{~cm}^{2}$ via 3-5 septocutaneous perforators. The other branch from the DPB was noted to travel radialward and distally. Interestingly, it anastomosed with the 1, 2 intercompartmental supraretinacular artery (1, 2 ICSRA $)^{14}$ from the radial artery in 6 of 6 specimens where this branch was traced ( - Figs. 3, 4). - Fig. 5 is a diagrammatic representation of the relationship of the DPB and 1, 2 ICSRA.

Preserved cadaveric specimens provided useful information for correlation with the fresh tissue dissections. The India ink provided contrast, coloring arteries black. However, the quality of anatomic detail obtained in preserved cadavers was inferior to the results from lead oxide-injected fresh specimens.

\section{Clinical Cases}

\section{Case 1}

A 56-year-old male, known diabetic and hypothyroid on treatment, presented with chronic nonhealing ulcer of the tongue. Biopsy of the lesion was reported as moderately differentiated squamous cell carcinoma. He received neoadjuvant chemotherapy followed by right hemiglossectomy and free microvascular radial artery flap for reconstruction.

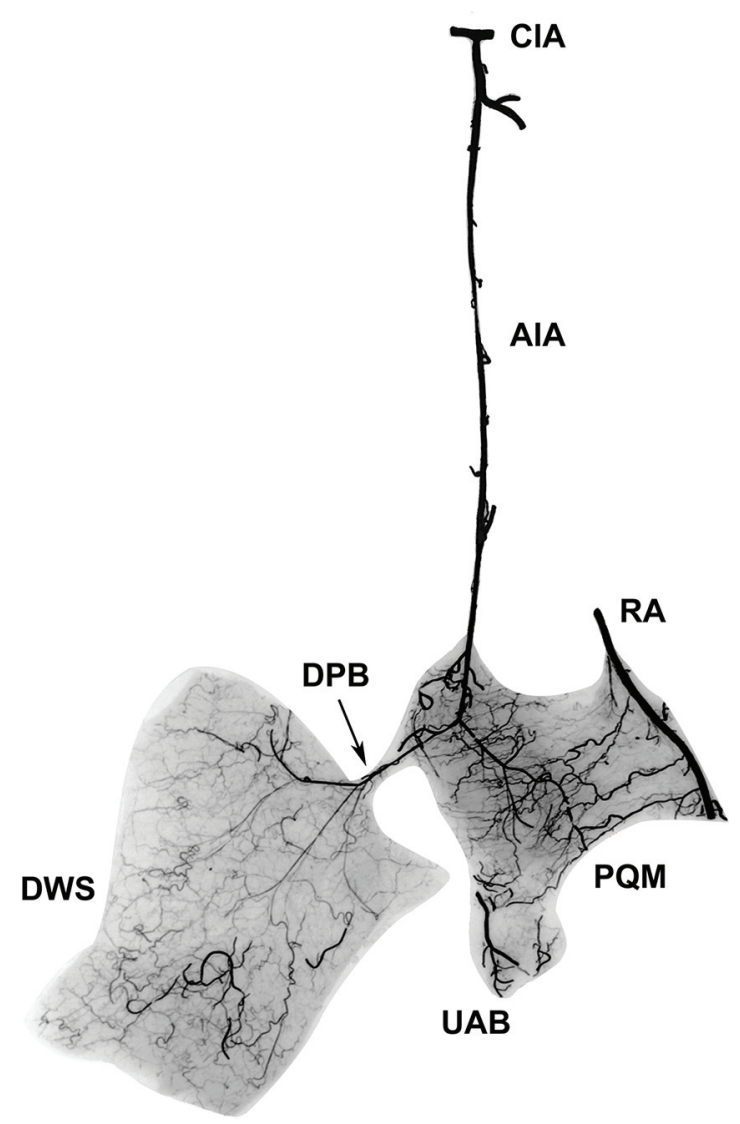

Fig. 3 Angiogram of the common interosseous artery (CIA) and anterior interosseous artery (AIA) from the same specimen as in Fig. 2. The intramuscular course of the AIA as well as contributions from the radial artery (RA) and ulnar artery (UA) are very well seen in the pronator quadratus muscle (PQM) on lead oxide-gelatin injection. The dorsal cutaneous perforating branch (DPB) supplying the skin on the dorsum is shown by the arrow.



Fig. 4 Photograph of a dissection specimen of an injected fresh cadaver showing the dorsal cutaneous perforating branch (DPB) of the anterior interosseous artery (AIA) and the 1, 2 intercompartmental supraretinacular artery (1, 2 ICSRA). The Listers tubercle (LT) is marked for correlation. The arterial branches supplying the skin paddle from the DPB can also be seen. 
The flap donor site in the left distal forearm broke down and became an infected ulcer (-Fig. 6 a). Following debridement and excision of exposed and necrotic flexor carpi radialis tendon, a pedicled PQM flap was raised and the defect with the exposed flexor tendons and median nerve was covered ( - Fig. 6 b, c). A split-thickness skin graft (STSG) was applied over the muscle. The flap healed well ( - Fig. 6 d). Following healing, he received further adjuvant chemotherapy.

\section{Case 2}

A 57-year-old lady with infiltrative ductal carcinoma in left breast and axillary lymphadenopathy underwent chemoport insertion and developed a nonhealing ulcer on the left wrist due to anthracycline extravasation. Pus culture from the wound grew Staphylococcus aureus sensitive to Cloxacillin.

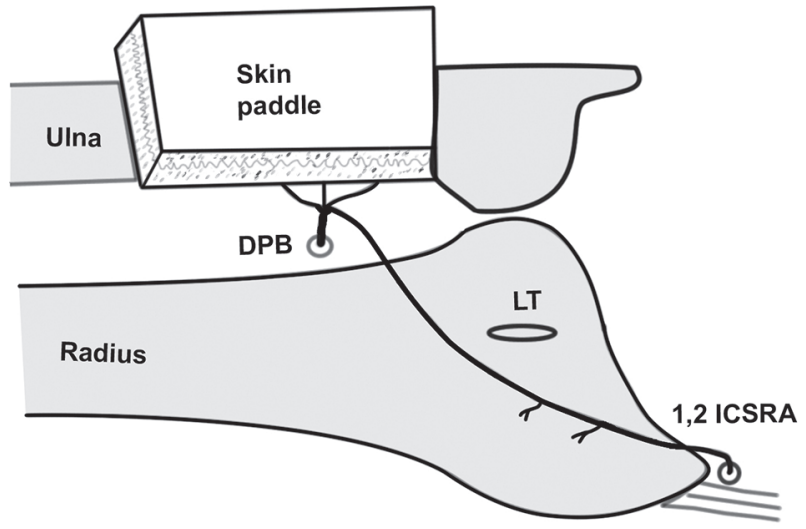

EPB/ APL

Fig. 5 Schematic representation of Fig. 3 showing the relation between the dorsal cutaneous perforating branch (DPB) of the anterior interosseous artery (AIA) and the 1, 2 intercompartmental supraretinacular artery (1, 2 ICSRA). The 1st extensor compartment tendons (extensor pollicis brevis/abductor pollicis longus [EPB/APL]) and Listers tubercle (LT) are marked.
She was treated with antibiotics and serial wound dressings. However, the ulcer failed to heal ( - Fig. 7 a). She underwent ulcer debridement, flexor tenosynovectomy, excision of flexor carpi radialis and palmaris longus muscles, followed by pedicled PQM flap to cover the defect ( $\sim$ Fig. 7 b, c). A STSG was applied over the muscle in this case also ( - Fig. 7 d). She then underwent modified radical mastectomy and postop chemotherapy for her breast cancer.

\section{Discussion}

The PQ is a quadrilateral muscle belonging to its own distinct forearm space in the deep volar compartment of the forearm. ${ }^{15}$ It is composed of a superficial and deep head with an occasional dorsal head. ${ }^{16}$ In our dissections, the superficial and deep heads were consistently seen. The dorsal head could not be identified in these specimens.

Basmajian ${ }^{17}$ suggested that PQ is the main forearm pronator based on his electromyography studies. He found that the pronator teres is a reinforcing muscle when rapid pronation or pronation against resistance is required. However, due to the presence of pronator teres, PQ is considered expendable and may be removed without obvious weakness of pronation. ${ }^{3}$ The role of PQ in stabilizing the ulna and in radioulnar convergence was investigated. ${ }^{16,18}$ The amplitude of motion and other physical characteristics of the PQ have been well-studied. ${ }^{19,20}$ Brand has reported the resting fiber length of $3.0 \mathrm{~cm}$, mass fraction of $1.8 \%$, and tension fraction of $3.0 \%$ for the PQ. ${ }^{19}$

Previous reports suggest that the PQ receives its primary blood supply consistently from the AIA. ${ }^{1}$ This was confirmed in our dissections. It also received minor pedicles from RA in all specimens and from UA in $77 \%$ of specimens. Hence, it can be classified as a type II muscle based on the classification of Mathes and Nahai (one dominant pedicle and two minor pedicles). ${ }^{21} \mathrm{Few}$ studies have focused on the intramuscular vascular anatomy of the PQM. Salmon performed lead oxide


Fig. 6 (a). Patient with moderately differentiated squamous cell carcinoma of tongue had right hemiglossectomy and free microvascular radial artery flap with flap donor site breakdown exposing median nerve. (b). The wound is debrided, and the pronator quadratus PQ flap is elevated based on the AIA. (c). A split-thickness skin graft (STSG) applied over the muscle flap. (d). The wound healed well on 3 months follow-up. 

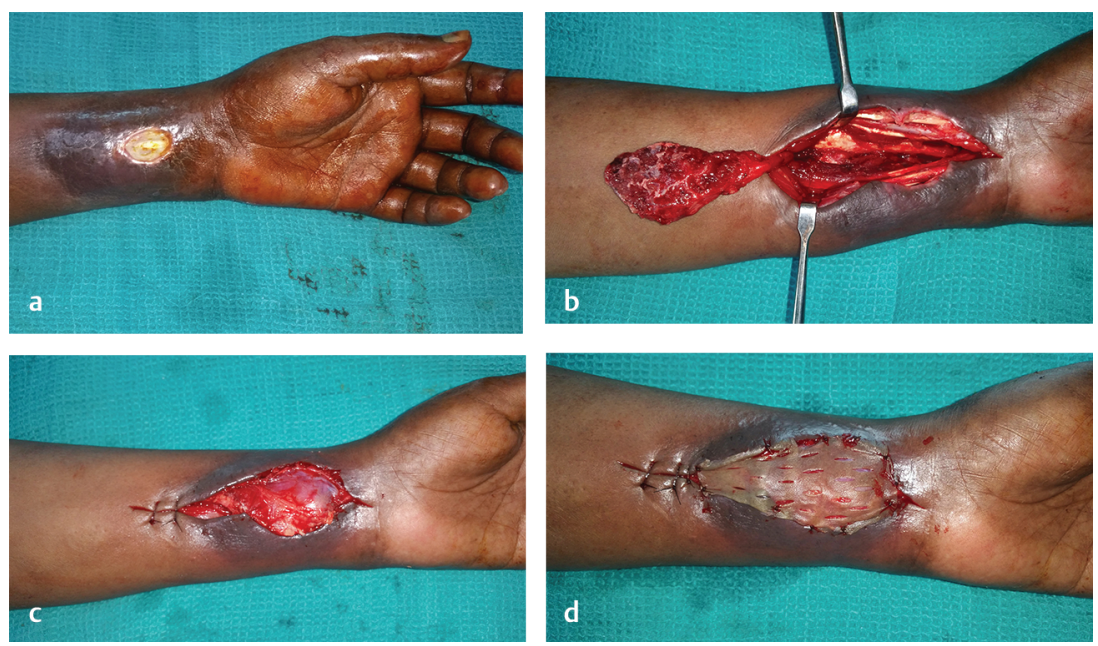

Fig. 7 (a). Patient with infiltrative ductal carcinoma breast had nonhealing ulcer over the left wrist due to anthracycline extravasation. (b). Following debridement, a pedicled pronator quadratus muscle (PQM) flap is raised. (c). The PQM is then inset and used to cover the defect. (d). A split-thickness skin graft (STSG) was applied over the muscle in this case also.

injection studies in the 1930's and wrote his classic book on the arteries of the muscles of the extremities and trunk. ${ }^{12}$ He reported that the PQM receives its blood supply via the AIA through a series of transverse branches. We found that the muscle is very well-vascularized by the AIA, which supplied the deep head with 1-2 branches and then penetrated it to supply the superficial head with 3-4 branches. These branches anastomosed with branches from the radial and ulnar arteries within the muscle. This confirms that the PQ is a type II muscle flap and also explains how bone grafts from distal radius based on $\mathrm{PQ}$ is very vascular.

The DPB from the AIA has been utilized in the distal dorsal forearm flap. ${ }^{22}$ Four types of free flaps based on the AIA have been described: fasciocutaneous, osteocutaneous, neurovascular and muscle. ${ }^{23}$

In six dissections, the dorsal perforating branch was meticulously dissected to trace the DPB supply to the dorsal wrist. An interesting finding was the anastomosis between this branch and the 1, 2 ICSRA branch from the radial artery. This finding has not been reported previously. This was seen in all the six specimens where this branch was traced. It may be possible to harvest vascularized bone from the dorsal aspect of the distal radius, based on this branch from the DPB of the AIA.

This anatomical study confirms the consistent vascular supply of the PQ by the AI vessels. The PQ maybe classified as a type II muscle based on its vascular supply. The intramuscular vascular anatomy is recorded as well as the cutaneous supply over the dorsal wrist skin by its perforating branches. Our anatomical study supports the use of this muscle as a free muscle transfer for coverage of defects up to $5 \times 5.5 \mathrm{~cm}^{2}$ or for functioning muscle transfer based on the AI nerve. The muscle flap dissection is straightforward. The arc of rotation of the pedicle is approximately $6 \mathrm{~cm}$, which concurs with the description by Dellon et $\mathrm{al}^{3}$.Harvest of the skin paddle can increase the area covered by another $5 \times 5 \mathrm{~cm}^{2}$ but involves a more difficult dissection. PQ may also be used as a functioning free muscle transfer to provide thenar abduction or facial reanimation with minimal donor defect. A distally based PQ flap based on the dorsal branch of AIA has been described. ${ }^{1}$

The clinical cases highlight the use of the PQM flap in covering the defects in the distal forearm. The prime indication of this flap in our opinion is covering exposed median nerve and providing a soft-tissue cushion for painful neuromas following median nerve repair at distal forearm level. It is also useful to cover exposed flexor tendons in the distal forearm level when used as a pedicled flap. The harvest is quick and simple. There was no weakness of thumb flexion following the harvest in both patients. Since the vascular pedicle is medium-sized, it can very well be used as free muscle flap.

\section{Conclusion}

We have provided further anatomical clarification of the PQM flap and demonstrated its use in clinical scenarios. This muscle appears to be an expendable, easily dissected useful option, especially in hand and wrist surgery.

\section{Conflicts of Interest}

None declared.

\section{References}

1 Rath S, Hung LK, Leung PC. Vascular anatomy of the pronator quadratus muscle-bone flap: a justification for its use with a distally based blood supply. J Hand Surg Am 1990;15(4):630-636

2 Leung PC, Hung LK. Use of pronator quadratus bone flap in bony reconstruction around the wrist. J Hand Surg Am 1990;15(4):637-640

3 Dellon AL, Mackinnon SE. The pronator quadratus muscle flap. J Hand Surg Am 1984;9(3):423-427

4 Kawai H, Yamamoto K. Pronator quadratus pedicled bone graft for old scaphoid fractures. J Bone Joint Surg $\mathrm{Br}$ 1988;70(5):829-831

5 Lee JC, Lim J, Chacha PB. The anatomical basis of the vascularized pronator quadratus pedicled bone graft. J Hand Surg [Br] 1997;22(5):644-646

6 Dautel G, Merle M. Pronator quadratus free muscle flap for treatment of palmar defects. J Hand Surg [Br] 1993;18(5):576-578 
7 Marshall MP, Gutheil JP. Pronator quadratus flap for burn reconstruction. J Burn Care Rehabil 2004;25(6):519-522

8 Lee WPA, Idler RS. Functional transfer of pronator quadratus free flap for thenar muscle loss. J Reconstr Microsurg 1996;12(2):77-80

9 Quatra F, Romeo OM, Lowemberg D, Brooks D, Buncke HJ. Functional transplantation of the pronator quadratus muscle for facial reanimation. A small muscle for a small job. Presented at the Plastic Surgery Research Council 47th Annual Meeting 2002

10 Tang ML, Geddes CR, Yang D, Morris SF. Modified lead oxide gelatin technique for vascular studies. J Clin Anat. 2002;1:73-78

11 Salmon M, Arteres de la Peau. Paris: Masson et Cie; 1936

12 Salmon M, Arteries of the muscles of the extremities. P 89. In: Taylor GI, Razaboni RM, eds. Arteries of the Muscles of the Extremities and the Trunk. St Louis: Quality Medical Publishing; 1994

13 Rees MJ, Taylor GI. A simplified lead oxide cadaver injection technique. Plast Reconstr Surg 1986;77(1):141-145

14 Sheetz KK, Bishop AT, Berger RA. The arterial blood supply of the distal radius and ulna and its potential use in vascularized pedicled bone grafts. J Hand Surg Am 1995;20(6):902-914

15 Sotereanos DG, McCarthy DM, Towers JD, Britton CA, Herndon JH. The pronator quadratus: a distinct forearm space? J Hand Surg Am 1995;20(3):496-499
16 Johnson RK, Shrewsbury MM. The pronator quadratus in motions and in stabilization of the radius and ulna at the distal radioulnar joint. J Hand Surg Am 1976;1(3):205-209

17 Basmajian JV, DeLuca CJ, Muscles alive-Their Functions Revealed by Electromyography. 5th ed. Baltimore, Williams and Wilkins; 1985: 283

18 Sauerbier M, Berger RA, Fujita M, Hahn ME. Radioulnar convergence after distal ulna resection. Acta Orthop Scand 2003;74(4):420-428

19 Brand PW, Beach RB, Thompson DE. Relative tension and potential excursion of muscles in the forearm and hand. J Hand Surg Am 1981;6(3):209-219

20 Stuart PR. Pronator quadratus revisited. J Hand Surg [Br] 1996;21(6):714-722

21 Mathes SJ, Nahai F. Classification of the vascular anatomy of muscles: experimental and clinical correlation. Plast Reconstr Surg 1981;67(2):177-187

22 Syed SA, Zahir KS, Zink JR, Saglaam O, Restifo RJ, Thomson JG. Distal dorsal forearm flap. Ann Plast Surg 1997;38(4):396-403

23 Shibata M, Ogishyo N. Free flaps based on the anterior interosseous artery. Plast Reconstr Surg 1996;97(4):746-755 